Internist 2010 $\cdot 51: 431-432$

DOI 10.1007/s00108-010-2583-8

Online publiziert: 20. Februar 2010

(c) Springer-Verlag 2010

J. Schölmerich

Klinik und Poliklinik für Innere Medizin I, Klinikum der Universität Regensburg

\title{
Schnittstellen der Inneren Medizin mit benachbarten Fachgebieten
}

delt. Die Autoren machen deutlich, dass eine nach neurologischen Symptomen orientierte Analyse des klinischen Bilds oft die differenzialdiagnostische Einordnung der Ursachen erlaubt, wobei hier ein gemeinsames Vorgehen von Internisten und Neurologen erforderlich ist.

Die 2. Arbeit befasst sich mit den Einschränkungen der Operabilität durch bestehende internistische Erkrankungen, die mit zunehmendem Alter der operierten Patienten zunehmen. Risikoscores ebenso wie Leitlinien stehen diesbezüglich zur Verfügung und werden in dem Aufsatz dargestellt. Es wird aber auch deutlich gemacht, dass die Einschätzung der Operabilität individuell nach einer ausführlichen Anamnese und einer körperlichen sowie gegebenenfalls auch funktionellen Untersuchung erfolgen muss. Insbesondere Patienten, die unter einer eingeschränkten körperlichen Belastbarkeit und Begleiterkrankungen leiden, sind mit einem höheren Risiko behaftet: Hier sollte präoperativ eine Optimierung oder die Einleitung einer spezifischen medikamentösen Therapie erfolgen. Gerade die kardiale Funktion bedarf einer intensiven Klärung und gegebenenfalls präoperativen Optimierung.

\section{beit des Internisten geben:}

Die 1. Arbeit befasst sich mit Veränderungen des Zentralnervensystems bei inneren Erkrankungen. Für die gesamte Vielfalt werden Beschwerden und Zeichen der veränderten Funktion des ZNS, von Störungen des Bewusstseins und der Kognition über Kopfschmerzen, Schwindel, zerebrale Anfälle, Halbseitensyndrome und andere motorische Störungen abgehan- auch spezifische morphologische Untersuchungsmodalität für verschiedene Fragestellungen der Inneren Medizin erwiesen. Dies betrifft die Angiologie, die Kardiologie, die Rheumatologie und insbesondere die Gastroenterologie und Hepatologie. In der Arbeit wird besonders die diagnostische Aussagekraft neuer MRTTechniken im Vergleich zu den bislang etablierten Verfahren Computertomographie und Sonographie analysiert.

Die 4. Übersicht beschreibt die Grenzen und Möglichkeiten der Biopsie in der Inneren Medizin. Hier werden exemplarisch der Gastrointestinaltrakt, das Herz, die Nieren und das Knochenmark abgehandelt. Verbesserte laborchemische, bildgebende und endoskopische Techniken haben die nicht-invasive bzw. makroskopische Diagnosesicherung deutlich verbessert und damit die Anzahl notwendiger Biopsien reduziert. Nach wie vor ist die Histopathologie aber in vielen Fällen Goldstandard der internistischen Diagnostik. Diese Entwicklungen werden exemplarisch anschaulich dargestellt und mit praktischen Hinweisen angereichert.

Der 5. Aufsatz beschreibt Symptome aus der Ophthalmologie als Hinweis auf internistische Erkrankungen. Hier wird deutlich, dass die ophthalmologischen Symptome oft die erste Manifestation eines generalisierten Leidens sind. Die Übersicht kann bei Weitem nicht alle Assoziationen ophthalmologischer Befunde mit internistischen Erkrankungen abdecken, sie macht aber exemplarisch an wichtigen Beispielen deutlich, wie wichtig eine enge und individuelle Kommunikation zwischen Augenarzt und Internisten ist, um 
eine optimale Betreuung der Patienten zu erreichen.

Die 6. Arbeit befasst sich mit dem Übergang von der pädiatrischen Betreuung, die stärker fürsorglich, kind- und familienzentriert geprägt ist, in die durch größere Autonomie und Eigenverantwortung geprägte Erwachsenenmedizin. Durch die Fortschritte der Medizin erreichen immer häufiger Kinder mit schweren chronischen Erkrankungen das Erwachsenenalter - ein klassisches Beispiel ist die Mukoviszidose. Ein fundiertes Wissen über diese bislang fast ausschließlich pädiatrischen Krankheitsbilder und Kenntnisse über die körperlichen und psychischen Entwicklungsstufen der Jugendlichen müssen daher auch zur Weiterbildung eines Internisten gehören.

Die 6 Arbeiten in diesem Heft machen eindrücklich klar, dass die Innere Medizin über zahlreiche Schnittstellen zu benachbarten Fachgebieten verfügt und dass diese auch aktiv genutzt werden können und müssen. Ich hoffe, dass diese Arbeiten den Lesern dies deutlich machen und für die tägliche Arbeit in Klinik und Praxis hilfreich sind.

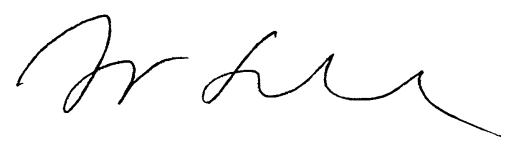

Prof. Dr. Jürgen Schölmerich

\section{Korrespondenzadresse \\ Prof. Dr. J. Schölmerich}

Klinik und Poliklinik für Innere Medizin I, Klinikum der Universität Regensburg 93042 Regensburg juergen.schoelmerich@klinik.uni-regensburg.de

\section{Studienaufruf}

Neues Verfahren zur Behandlung von

Emphysematikern

Bisher stehen medikamentöse Maßnahmen bei der Behandlung von COPD und Lungenemphysemen im Vordergrund.

Nach Ausschöpfung aller konservativen Optionen kommen bei einzelnen Patienten auch chirurgische Verfahren der Lungenvolumenreduktion zur Anwendung. Allerdings kann bei den meisten Patienten aufgrund der hohen Morbidität und Mortalität nicht zu einer Operation geraten werden. Nicht-chirugische endoskopische Maßnahmen sind daher als weniger riskante Alternativen entwickelt worden. Diese Methoden haben aber bisher keine signifikante Rolle bei der Behandlung dieser Patienten gespielt, da sie, aufgrund der häufig bei Emphysematikern vorliegenden kollateralen Ventilation, eine limitierte Effektivität zeigten.

Eine neu entwickelte bronchoskopische Technik wird jetzt im Rahmen einer europäischen Studie an Patienten im fortgeschrittenem Stadium eines Lungenemphysems bewertet. In Deutschland nehmen die Thoraxkliniken in Heidelberg, Grosshansdorf, München, Bad Berka, Hemer und Berlin an dieser Studie teil und testen die neue Technik.

Die Funktionsstörung des Atmens bei Emphysematikern ist in erster Linie auf die Hyperinflation der Lunge zurückzuführen. Diese verursacht Atemnot und beschränkt die körperliche Leistungsfähigkeit. Um das Volumen der vergrößerten Bereiche der Lunge zu verringern, wird im Rahmen der Studie ein flüssiger Schaum über einen Katheter in die Lunge eingeführt. Dort polymerisiert dieser Schaum und versiegelt so die behandelte Lungenregion. Der bronchoskopische Eingriff dauert 10 bis 15 Minuten. Die Patienten werden nach dem Eingriff ein Jahr lang durch regelmäßige Nachuntersuchungen betreut. Bei den Nachuntersuchungen werden Lungenfunktionsmessungen und Blutuntersuchungen durchgeführt. Mit Fragebögen zu Symptomen und zur Lebensqualität wird die Behandlungen dokumentiert. Die Wirkung des Schaums ist dauerhaft; eine spätere Entfernung ist nicht möglich.
In Deutschland sind bisher 34 Patienten im Rahmen dieser Studie behandelt worden. Alle Behandlungen verliefen ohne Komplikationen. Nach der Behandlung stellte sich bei allen Patienten, als Reaktion auf den Schaumversiegler, eine kurzfristige, grippeartige Verschlechterung des Gesundheitszustandes ein. Diese Symptome konnten problemlos mit den üblichen Medikamenten behandelt werden. Einige der Patienten haben bereits ihre Nachuntersuchung abgeschlossen. Bei der Mehrzahl der Patienten zeigten sich Hinweise auf eine Lungenvolumenreduktion mit Verbesserungen der Lungenfunktion und damit auch der Lebensqualität.

Die Zahl der betroffenen Emphysematiker, denen mit dieser Behandlung geholfen werden könnte, ist weitaus höher. Weitere Patienten mit fortgeschrittenem heterogenen und homogenen Emphysem können bei Erfüllung der Ein- und Ausschlusskriterien in die Studie aufgenommen werden.

Informationen zur Studie und zu den Ein- und Ausschlusskriterien sowie die Kontakte der einzelnen Kliniken können bei der Thoraxklinik Heidelberg angefragt werden.

Thoraxklinik am Universitätsklinikum Heidelberg

Tel.: +49-6221-396-1200

Mail: martina.mundelsee@ thoraxklinik-heidelberg.de 